\title{
PFKFB4 as a promising biomarker to predict a poor prognosis in patients with gastric cancer
}

\author{
FANG WANG ${ }^{1 *}$, XIAOTING WU ${ }^{1,2^{*}}$, YAJUN LI $^{1}$, XIANGMEI CAO ${ }^{3}, \mathrm{CAO} \mathrm{ZHANG}^{4}$ and YUJING GAO ${ }^{2}$ \\ ${ }^{1}$ Department of Gastroenterology, General Hospital of Ningxia Medical University, Ningxia Medical University; \\ ${ }^{2}$ Key Laboratory of Fertility Preservation and Maintenance of Ministry of Education, Department of \\ Biochemistry and Molecular Biology, Ningxia Medical University; ${ }^{3}$ Department of Pathology, School of \\ Basic Medical Sciences, Ningxia Medical University; ${ }^{4}$ Department of General Surgery, General Hospital of \\ Ningxia Medical University, Ningxia Medical University, Yinchuan, Ningxia 750004, P.R. China
}

Received June 28, 2020; Accepted December 14, 2020

DOI: $10.3892 / \mathrm{ol} .2021 .12557$

\begin{abstract}
Gastric cancer (GC) is one of the most common types of cancer worldwide. Previous studies have reported that phosphofructo-2-kinase/fructose-2,6-biphosphatase 4 (PFKFB4) functions as an oncoprotein in various types of cancer. However, the association between PFKFB4 and GC remains unclear. The present study analyzed the expression levels of PFKFB4 in 148 GC tissue samples, including 46 tumor tissues with matched adjacent normal tissues, using immunohistochemistry, compared the expression levels of PFKFB4 between GC and adjacent normal tissues, and determined the association between PFKFB4 expression levels and patient clinicopathological characteristics. In addition, survival curves were generated using the Kaplan-Meier (KM) plotter database to evaluate the association between PFKFB4 expression and GC prognosis. The results revealed that PFKFB4 expression was upregulated in GC tissues compared with in adjacent normal tissues. PFKFB4 expression was associated with patient age, tumor size, pathological tumor (pT) stage and tumor-node-metastasis (pTNM) stage, and upregulated expression levels of PFKFB4 were observed in tumor tissues from patients $<65$ years old (compared with that in patients $\geq 65$ years old), as well as patients with a larger tumor size and an advanced stage (pT and pTNM stage) disease. In addition, KM survival analysis demonstrated that patients with low PFKFB4 expression had a significantly improved overall
\end{abstract}

Correspondence to: Dr Yujing Gao, Key Laboratory of Fertility Preservation and Maintenance of Ministry of Education, Department of Biochemistry and Molecular Biology, Ningxia Medical University, 1160 Shengli Street, Xingqing, Yinchuan, Ningxia 750004, P.R. China

E-mail: gaoyujing2004@126.com

*Contributed equally

Key words: phosphofructo-2-kinase/fructose-2,6-biphosphatase 4, protein expression, gastric cancer, clinicopathological characteristics, prognosis survival (OS), first progression survival and post-progression survival times compared with those with high PFKFB4 expression. Furthermore, PFKFB4 expression was negatively associated with OS time in patients with late pT and pTNM stage disease. In conclusion, the results of the present study indicated that the upregulated PFKFB4 expression in GC tissues may serve as a biomarker for a more advanced disease and a poor prognosis in patients with GC.

\section{Introduction}

In recent years, malignant tumors have become a major public health burden that significantly impact the health of individuals worldwide. Gastric cancer (GC) is a cancer of the gastrointestinal tract that currently ranks as the 5th most common type of cancer worldwide, accounting for an estimated 1,033,000 new cases and 783,000 deaths in 2018 (1). Due to the low early diagnosis rate of GC and the poor therapeutic effects of traditional treatment regimens, which usually combine surgery and chemotherapy, the prognosis of patients with GC remains poor. The Tumor-Node-Metastasis (TNM) classification, which classifies tumors according to the pathological examination of resected specimens, remains the most reliable tool for predicting the clinical outcome of patients with GC (2). However, substantial differences in survival are observed for patients at the same clinical stage $(2,3)$. Although numerous molecules have been reported as potential biomarkers for predicting clinical outcomes in GC, such as upregulated expression levels of SWI/SNF related, matrix associated, actin dependent regulator of chromatin, subfamily e, member 1 and chemokine receptor 6 , which were discovered to be associated with a poor prognosis in patients with GC $(4,5)$, none of the identified molecules have been clinically evaluated for the disease to date. Therefore, further studies are required to determine specific biomarkers that may help distinguish patients with different prognoses more accurately.

It is well known that cancercells prefer to metabolize glucose via glycolysis rather than mitochondrial oxidative phosphorylation, even in oxygen-rich conditions, and this phenomenon is referred to as the Warburg effect or aerobic glycolysis (6). Phosphofructo-2-kinase/fructose-2,6-biphosphatase 4 
(PFKFB4) is one of the four subtypes of the bifunctionalenzyme PFKFB (7). By regulating the levels of fructose-2,6-bisphosphate, the strongest allosteric activator of phosphoric acid fructose kinase-1, PFKFB4 serves an important role in modulating the glycolytic and pentose phosphate pathway flux, in addition to the production of ATP, which affects the growth of tumors $(8,9)$. Recently, a previous study conducted in prostate cancer revealed that CD44 could modulate the aggressive phenotype of prostate cancer cells by regulating the expression levels of pyruvate dehydrogenase kinase, isozyme 1 and PFKFB4 (10). In addition, another previous study reported that PFKFB4 enhanced the migration and invasion of GC cells by downregulating the expression levels of the tumor suppressor gene liver kinase B1 (LKB1) (11), indicating an association between PFKFB4 and cancer development. However, to the best of our knowledge, the expression levels and clinical significance of PFKFB4 in GC remain to be elucidated.

The present study analysed PFKFB4 expression in GC and adjacent normal tissues using immunohistochemistry (IHC), and further determined the association between PFKFB4 expression levels and the clinicopathological characteristics of patients with GC. In addition, the association between PFKFB4 expression levels and the survival of patients with GC was investigated using the Kaplan-Meier (KM) plotter database.

\section{Materials and methods}

Patients and specimens. A total of $148 \mathrm{GC}$ tissues were collected from patients diagnosed with GC at the General Hospital of Ningxia Medical University (Yinchuan, China) between September 2012 and December 2013. There were only $46 \mathrm{GC}$ tissues which contained matched adjacent normal tissues ( $2 \mathrm{~cm}$ from the edge of tumors) due to the process of generating the pathological sections. Chemotherapy, radiotherapy and other antitumor treatments were not administered to the patients before surgery. Oral informed consent was obtained from all patients prior to collecting the specimens. The study was approved by the Medical Research Ethics Review Committee of The General Hospital of Ningxia Medical University. The specimens were fixed with $4 \%$ paraformaldehyde for $24 \mathrm{~h}$ at room temperature, embedded in paraffin and cut into $5-\mu \mathrm{m}$-thick sections for IHC analysis. The pathological diagnosis was performed by two senior pathologists according to the hematoxylin and eosin staining results. TNM stage evaluation was determined according to the criteria indicated in the staging procedures of the International Association for the study of GC (12). The clinicopathological data of the patients are summarized in Table I. It should be noted that 2/148 GC cases had no information regarding the tumor differentiation degree.

IHC staining. The expression levels of PFKFB4 in GC tissues were determined by IHC analysis. Briefly, the formalin-fixed and paraffin-embedded tissue sections were heated at $65^{\circ} \mathrm{C}$ for $1 \mathrm{~h}$, then deparaffinized in xylene and rehydrated in a series of graded ethanol. After washing 3 times with PBS, the sections were incubated with $3 \%$ hydrogen peroxide at room temperature for $10 \mathrm{~min}$ to block endogenous peroxidase activity. EDTA buffer ( $\mathrm{pH}$ 8.0) was then used for antigen retrieval.
Subsequently, the sections were blocked with $10 \%$ goat serum (cat. no. ZLI-9022; OriGene Technologies) for $10 \mathrm{~min}$ at $37^{\circ} \mathrm{C}$ prior to incubation with an anti-PFKFB4 antibody (1:100; cat. no. ab137785; Abcam) at $37^{\circ} \mathrm{C}$ for $1 \mathrm{~h}$ and then overnight at $4^{\circ} \mathrm{C}$ in a humidified chamber. Following primary antibody incubation, the sections were washed 3 times with PBS and then incubated with an HRP-conjugated peroxidase working solution (cat. no. P0448; Dako; Agilent Technologies, Inc.) for $30 \mathrm{~min}$ at $37^{\circ} \mathrm{C}$. The sections were incubated with the chromogen substrate $3,3^{\prime}$-diaminobenzidine for $10 \mathrm{~min}$ at room temperature, followed by haematoxylin counterstaining at room temperature for $1 \mathrm{~min}$. Finally, the slides were dehydrated in a graded series of alcohol and xylene, and sealed with neutral balsam and a glass coverslip.

The expression levels of PFKFB4 were observed and the images were captured using a light microscope at x100 and x400 magnification, and determined using a semi-quantitative method (13). The immunoreactivity score was evaluated blindly by two senior pathologists. The staining intensity was scored as follows: 0 , negative; $1+$, light yellow; $2+$, yellowish brown; and $3+$, brown. The number of stained cells was also scored and divided into four groups according to the percentage of positively stained cells: 0 , no positively stained cells; $1+, \leq 10 \%$ positively stained cells; $2+, 11-50 \%$ positively stained cells; $3+, 51-75 \%$ positively stained cells; and $4+,>75 \%$ positively stained cells. The final score was the product of the staining intensity score and the score for the percentage of positively stained cells. The median immunoreactivity score was used as the cut-off value to define high and low expression in the samples.

Survival analysis. KM survival analysis for GC and the subsequent log-rank test were performed using the KM plotter database (http://kmplot.com/analysis/index. php? $\mathrm{p}=$ service \&cancer=gastric). Briefly, Affy ID 206246_at (PFKFB4) was selected for survival analysis. A total of 875 patients with GC were included for overall survival (OS; the survival time from diagnosis until death from any cause) analysis, while 640 patients were used to analyze the first progression survival (FPS; the survival time without progression of the disease), and 498 patients were used to analyze the post-progression survival (PPS; the survival time following progressive disease during treatment) times. In addition, 241 patients with GC with pT2 stage disease, 204 patients with pT3 stage disease, 140 patients with stage II disease, 305 patients with stage III disease and 148 patients with stage IV disease were used to determine the association between PFKFB4 expression and OS.

Statistical analysis. Statistical analyses were performed using SPSS version 17.0 software (SPSS, Inc.). A Mann-Whitney U test was performed to compare the differences in the expression levels of PFKFB4 between the GC and adjacent normal tissues. The statistical differences between the 46 pairs of GC and matched adjacent normal tissues were determined using a Wilcoxon signed rank test. Scatterplots showing the distribution of PFKFB4 staining scores in GC and adjacent normal tissues were drawn using GraphPad Prism V5.01 software (GraphPad Software, Inc.). The association between PFKFB4 expression and the clinicopathological characteristics of the 
Table I. PFKFB4 expression and clinicopathological characteristics of patients with gastric cancer $(n=148)$.

\begin{tabular}{|c|c|c|c|c|c|}
\hline \multirow[b]{2}{*}{ Variables } & \multirow[b]{2}{*}{ Cases, $\mathrm{n}$} & \multicolumn{2}{|c|}{ PFKFB4 expression } & \multirow[b]{2}{*}{$\chi^{2}$} & \multirow[b]{2}{*}{ P-value } \\
\hline & & Low, n (\%) & High, n (\%) & & \\
\hline \multicolumn{6}{|l|}{ Age, years } \\
\hline$\geq 65$ & 57 & $42(73.7)$ & $15(26.3)$ & \multirow[t]{2}{*}{7.782} & \multirow[t]{2}{*}{$0.005^{\mathrm{a}}$} \\
\hline$<65$ & 91 & $46(50.5)$ & $45(49.5)$ & & \\
\hline \multicolumn{6}{|l|}{ Sex } \\
\hline Male & 123 & $71(57.7)$ & $52(42.3)$ & \multirow[t]{2}{*}{0.910} & \multirow[t]{2}{*}{0.340} \\
\hline Female & 25 & $17(68.0)$ & $8(32.0)$ & & \\
\hline \multicolumn{6}{|c|}{ Differentiation } \\
\hline Well & 21 & $10(47.6)$ & $11(52.4)$ & \multirow[t]{4}{*}{1.940} & \multirow[t]{4}{*}{0.379} \\
\hline Moderate & 22 & $15(68.2)$ & $7(31.8)$ & & \\
\hline Poor & 103 & $62(60.2)$ & $41(39.8)$ & & \\
\hline NA & 2 & $1(50.0)$ & $1(50.0)$ & & \\
\hline \multicolumn{6}{|c|}{ Tumor size, $\mathrm{cm}$} \\
\hline$\geq 4$ & 88 & $46(52.3)$ & $42(47.7)$ & \multirow[t]{2}{*}{4.651} & \multirow[t]{2}{*}{$0.031^{\mathrm{a}}$} \\
\hline$<4$ & 60 & $42(70.0)$ & $18(30.0)$ & & \\
\hline \multicolumn{6}{|l|}{ pT stage } \\
\hline $\mathrm{T} 1-2$ & 39 & $31(79.5)$ & $8(20.5)$ & \multirow[t]{2}{*}{8.812} & \multirow[t]{2}{*}{$0.003^{\mathrm{a}}$} \\
\hline T3-4 & 109 & $57(52.3)$ & $52(47.7)$ & & \\
\hline \multicolumn{6}{|l|}{ pN stage } \\
\hline N0 & 59 & $40(67.8)$ & $19(32.2)$ & \multirow[t]{2}{*}{2.829} & \multirow[t]{2}{*}{0.093} \\
\hline N1-3 & 89 & $48(53.9)$ & $41(46.1)$ & & \\
\hline \multicolumn{6}{|l|}{ pM stage } \\
\hline M0 & 141 & $85(60.3)$ & $56(39.7)$ & & \multirow[t]{2}{*}{$0.442^{\mathrm{b}}$} \\
\hline M1 & 7 & $3(42.9)$ & $4(57.1)$ & & \\
\hline \multicolumn{6}{|c|}{ pTNM stage } \\
\hline $\mathrm{I}+\mathrm{II}$ & 45 & $33(73.3)$ & $12(26.7)$ & \multirow[t]{2}{*}{5.163} & \multirow[t]{2}{*}{$0.023^{\mathrm{a}}$} \\
\hline III+IV & 103 & $55(53.4)$ & $48(46.6)$ & & \\
\hline
\end{tabular}

${ }^{\mathrm{a}} \mathrm{P}<0.05$; ${ }^{\mathrm{b}}$ Fisher's exact test. NA, not applicable; $\mathrm{p}$, pathological; TNM, Tumor-Node-Metastasis; PFKFB4, phosphofructo-2-kinase/fructose-2,6-biphosphatase 4 .

patients was analysed using a $\chi^{2}$ test or Fisher's exact test. $\mathrm{P}<0.05$ was considered to indicate a statistically significant difference.

\section{Results}

Clinicopathological characteristics of patients with GC. In total, $148 \mathrm{GC}$ tissues, including $46 \mathrm{GC}$ and matched adjacent normal tissues, were collected to analyze the expression levels of PFKFB4. Among these specimens, 123 cases were men and 25 cases were women, with an age range of 23-96 years old and a mean age of $61.80 \pm 14.11$ years old. The detailed clinicopathological characteristics of the patients, including tumor differentiation, tumor size and TNM stage, are summarized in Table I.

Expression levels of PFKFB4 in GC and adjacent normal tissues. The expression levels of PFKFB4 in GC tissues were determined using IHC. Positive PFKFB4 expression was observed in almost all the tumor and adjacent normal tissues. Fig. 1 shows the representative IHC staining images of PFKFB4 expression in GC tissues. PFKFB4 was found to be mainly expressed in the cytoplasm and nucleus of the cells, and distributed diffusely throughout the tumor tissues. The intensity and range of PFKFB4 expression was stronger in the cytoplasm compared with the nucleus. However, the expression levels of PFKFB4 were markedly upregulated in the cell nuclei of tumor tissues with high PFKFB4 expression. Following the semi-quantification, PFKFB4 expression between GC and normal adjacent normal tissues was compared. The results revealed that PFKFB4 expression was significantly upregulated in the tumor tissues compared with in the adjacent normal tissues (median, 4 vs. 3 ; $\mathrm{P}=0.0002$; Fig. 2A). In addition, in the matched 46 pairs of GC and adjacent normal tissues, PFKFB4 expression was significantly upregulated in the GC tissues compared with in the normal tissues $(\mathrm{P}=0.015$; Fig. $2 \mathrm{~B})$. 

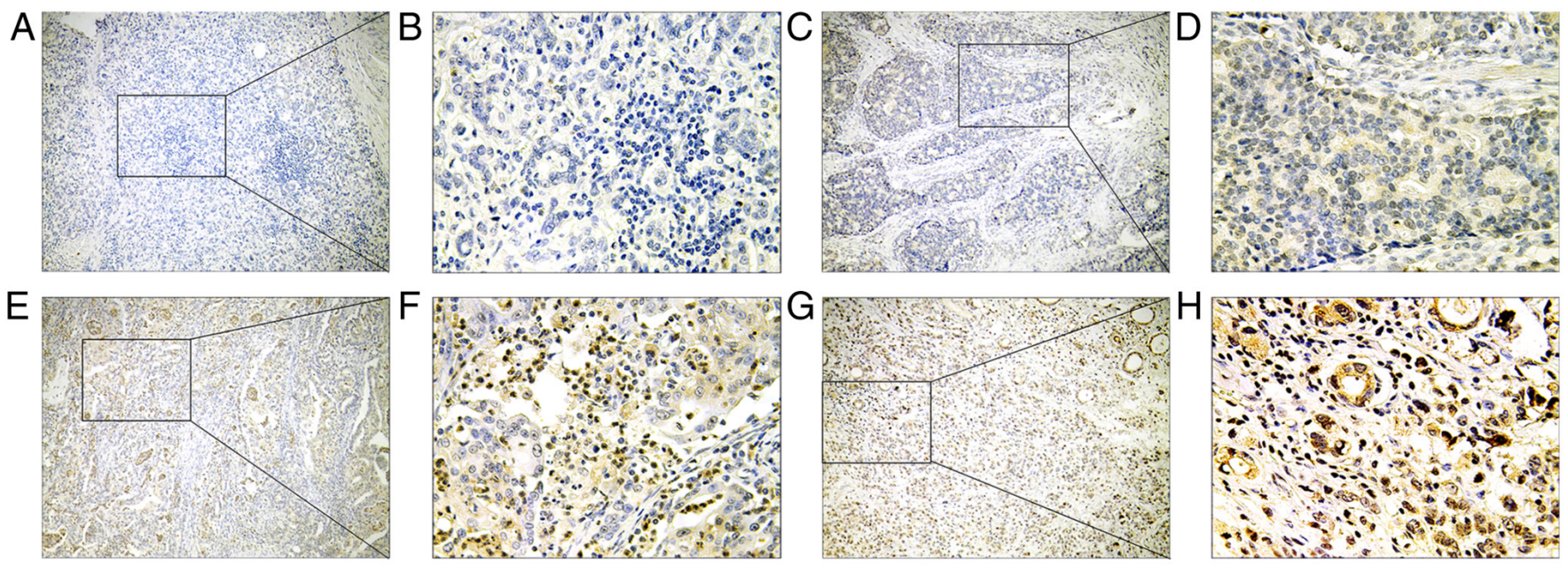

Figure 1. Representative immunohistochemistry images of phosphofructo-2-kinase/fructose-2,6-biphosphatase 4 expression in gastric cancer tissues. Negative staining at magnifications (A) x100 and (B) x400. Weak staining at magnifications (C) x100 and (D) x400. Moderate staining at magnifications (E) x100 and (F) $\mathrm{x} 400$. Strong positive staining at magnifications $(\mathrm{G}) \times 100$ and $(\mathrm{H}) \times 400$.

A



B

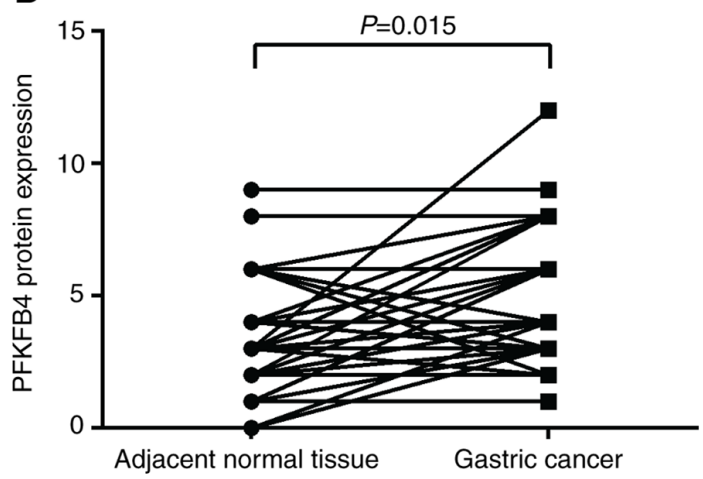

Figure 2. PFKFB4 expression is upregulated in GC tissues compared with in adjacent normal tissues. (A) Distribution of PFKFB4 staining scores in adjacent normal tissues $(\mathrm{n}=46)$ and $\mathrm{GC}$ tissues $(\mathrm{n}=148)$ was presented as the median with interquartile range. The P-value was determined using the Mann-Whitney $\mathrm{U}$ test. (B) Comparison of PFKFB4 staining scores in 46 pairs of GC and matched adjacent normal tissues. The P-value was determined using a Wilcoxon signed rank test. PFKFB4, phosphofructo-2-kinase/fructose-2,6-biphosphatase 4; GC, gastric cancer.

Association between PFKFB4 expression and the clinicopathological characteristics of patients with GC. The association between PFKFB4 protein expression in GC tissues and the clinicopathological characteristics of patients was subsequently analyzed. According to the median immunoreactivity score of PFKFB4 expression in the tumor tissues, which was 4 , specimens with an immunoreactivity score $>4$ were defined as having high PFKFB4 expression, while those with an immunoreactivity score $<4$ were defined as having low PFKFB4 expression. As shown in Table I, 59.5\% (88/148) and $40.5 \%(60 / 148)$ of the cases were classified as low and high expression, respectively. The percentage of specimens with high PFKFB4 expression was markedly increased in the $<65$ age group compared with that in the $\geq 65$ age group (49.5 vs. 26.3\%; $\mathrm{P}=0.005$ ). The percentage of tumors with high PFKFB4 expression and a large tumor size $(\geq 4 \mathrm{~cm})$ compared with a smaller tumor size $(<4 \mathrm{~cm})$ was also increased $(47.7$ vs. $30 \%$; $\mathrm{P}=0.031)$. Furthermore, in the advanced pT stage (pT3 and 4) or pTNM stage groups, the percentage of specimens with high PFKFB4 expression was markedly increased compared with the early stage groups $(\mathrm{P}<0.05)$. The aforementioned results indicated that there was a significant association between PFKFB4 expression level and the age of the patient, tumor size, pT stage, and pTNM stage. However, no significant associations were identified between PFKFB4 expression and the sex, tumor differentiation or pathological $\mathrm{N}$ and $\mathrm{M}$ stages of the patients.

Association between PFKFB4 expression and the survival of patients with $G C$. To further evaluate the clinical significance of PFKFB4 in GC, the difference in the survival time between patients with GC with high and low PFKFB4 expression was investigated using the online KM plotter database. As shown in Fig. 3, patients with high expression levels of PFKFB4 had a higher probability of a shorter OS, FPS and PPS compared with patients with low expression levels. The median survival time for OS, FPS and PPS was 22.5, 15.1 and 5.4 months, respectively, in the high PFKFB4 expression group, while it was 45.4, 71.7 and 12.1 months, respectively, in the low PFKFB4 expression group (Fig. 3). Considering that the 

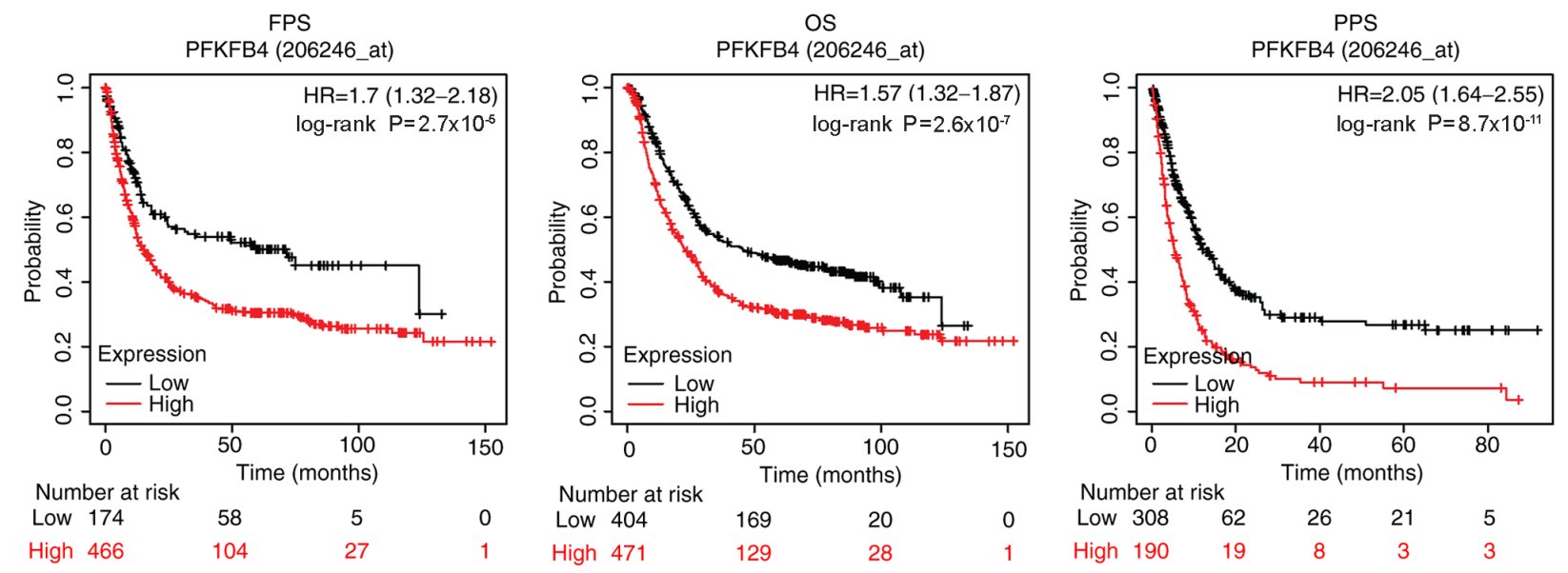

Figure 3. High PFKFB4 expression is positively associated with poor OS, FPS and PPS in patients with GC. Kaplan-Meier survival analysis of patients with GC and a subsequent log-rank test were performed using the Kaplan-Meier plotter database. OS, overall survival; FPS, first-progression survival; PPS, post-progression survival; PFKFB4, phosphofructo-2-kinase/fructose-2,6-biphosphatase 4; GC, gastric cancer; HR, hazard ratio.
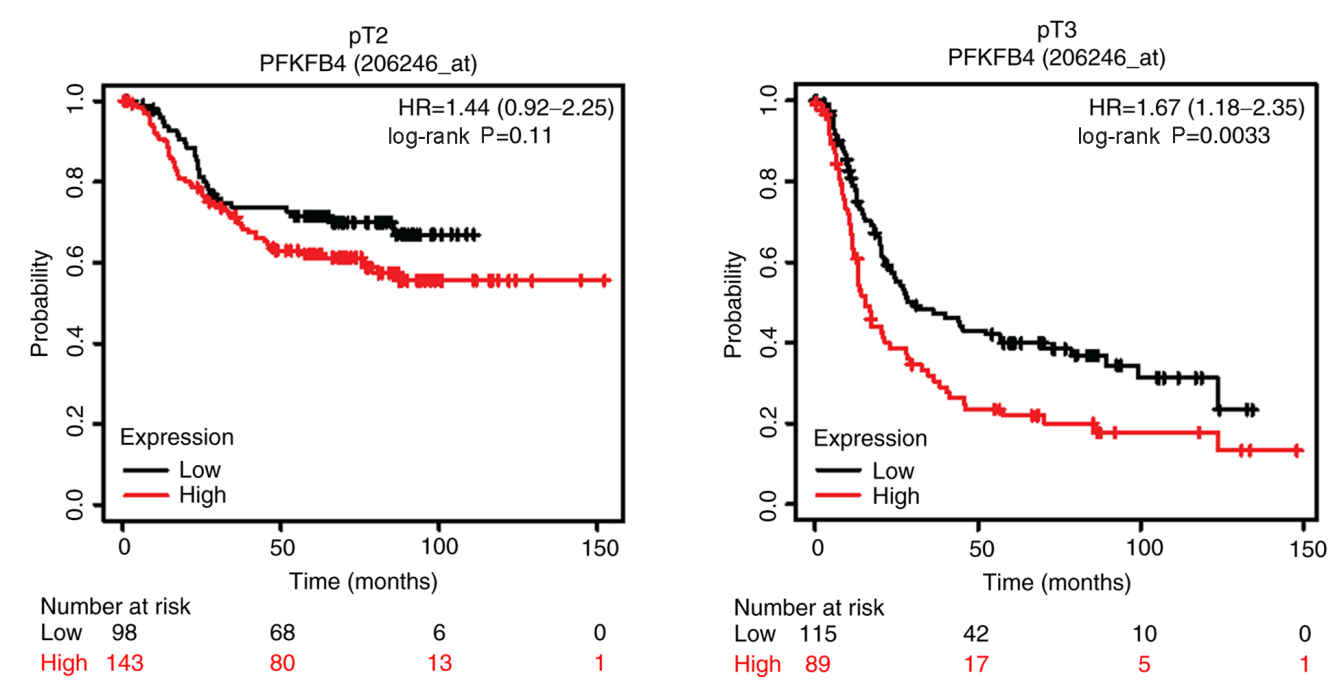

Figure 4. High PFKFB4 expression is associated with the OS of patients with GC with a high pT stage disease. Association between PFKFB4 expression and the OS of 241 patients with GC in the pT2 stage and 204 patients in the pT3 stage were analyzed using the Kaplan-Meier plotter database. The median OS time for patients with low and high PFKFB4 expression at pT2 stage disease was 30.9 and 26.4 months, respectively. The median OS time for patients with low and high PFKFB4 expression at pT3 stage disease was 30.4 and 15.3 months, respectively. OS, overall survival; pT, pathological tumor; PFKFB4, phosphofructo-2-kinase/fructose-2,6-biphosphatase 4; GC, gastric cancer; HR, hazard ratio.

expression levels of PFKFB4 were upregulated in advanced stage tumor tissues, the association between PFKFB4 expression and the OS of patients with different tumor stages was further analysed. The results revealed that PFKFB4 expression was not significantly associated with OS in patients with an early pT stage (pT2) or TNM stage (stage II) disease. The median OS times for patients with low and high PFKFB4 expression at pT2 disease stage was 30.9 and 26.4 months respectively, while the OS time in patients with low and high PFKFB4 expression at stage II disease was 78.6 and 35.2 months respectively. However, at the advanced stages (pT3 and TNM stages III and IV), high expression levels of PFKFB4 were significantly associated with a poor survival in patients with GC (Figs. 4 and 5). The median OS time for patients with low and high PFKFB4 expression at pT3 disease stage was 30.4 and 15.3 months respectively. Furthermore, OS time was 52.6 and 25.17 months, respectively for patients with low and high expression of PFKFB4 at stage III disease, and 20.03 and 11.47 months for patients at stage IV disease, respectively. Collectively, these results indicated that PFKFB4 may be a predictor for a poor prognosis in patients with GC.

\section{Discussion}

Several previous studies have reported that PFKFB4 functions as an oncoprotein in various types of cancer, including breast (14), prostate (15), bladder (16), hepatocellular carcinoma and thyroid cancer (17). In addition, a previous study demonstrated that 5-(n-(8-methoxy-4-quinolyl)amino) pentyl nitrate, a selective inhibitor of PFKFB4, suppressed the glycolysis and proliferation of multiple human cancer cell lines (18). PFKFB4 is a bi-functional metabolic enzyme that synthesizes a potent allosteric activator for one of the rate-limiting enzymes of glycolysis. Thus, PFKFB4 is able to modulate glucose 

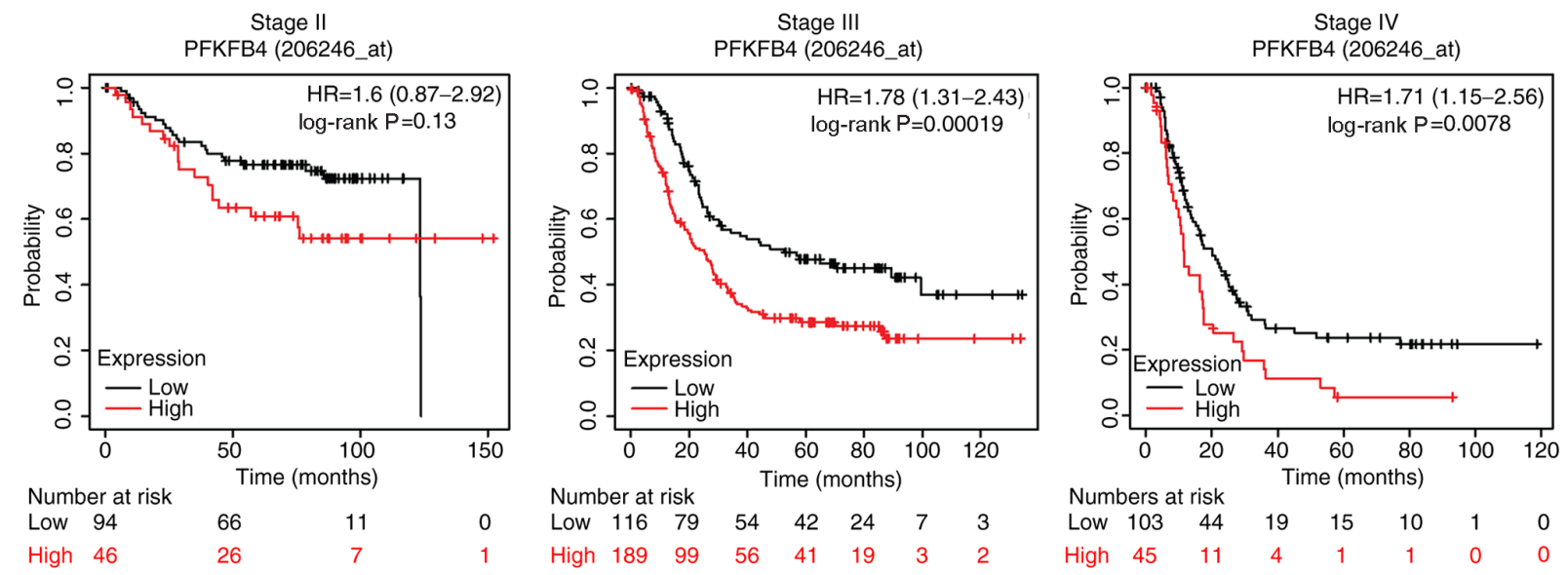

Figure 5. High PFKFB4 expression is associated with the OS of patients with GC with a high pTNM stage disease. Association between PFKFB4 expression and the OS of 140 patients with GC with stage II disease, 305 patients with stage III disease and 148 patients with stage IV disease were analyzed using the Kaplan-Meier plotter database. The median OS time for patients with low and high PFKFB4 expression at stage II disease was 78.6 and 35.2 months, respectively. The median OS time for patients with low and high expression of PFKFB4 at stage III disease was 52.6 and 25.17 months, respectively. The median OS time for patients with low and high PFKFB4 expression at stage IV disease was 20.03 and 11.47 months, respectively. OS, overall survival; pTNM, pathological Tumor-Node-Metastasis; PFKFB4, phosphofructo-2-kinase/fructose-2,6-biphosphatase 4; GC, gastric cancer; HR, hazard ratio.

metabolism, which is reprogrammed in cancer cells (19). One of the mechanisms underlying the tumor-promoting effect of PFKFB4 is its ability to regulate the glycolytic flux under both normoxic and hypoxic conditions (10,16,20-23). In addition, PFKFB4 has been discovered to promote tumor progression through glycolysis-independent mechanisms $(14,17,24,25)$. For example, it has been previously reported that PFKFB4 modulates the chemoresistance of small-cell lung cancer by regulating autophagy (24). PFKFB4 promotes breast cancer tumorigenesis by phosphorylating and activating steroid receptor coactivator-3, a crucial transcriptional coactivator of the estrogen receptor (25). PFKFB4 also induces the production of hyaluronan by activating p38 signaling (14). However, whether PFKFB4 is implicated in the tumorigenesis of GC remains unclear. Only one study demonstrated that PFKFB4 promotes the migration and invasion of GC cells by phosphorylating SRC-2 and inhibiting LKB1 expression (11).

The present study provided evidence to suggest an association between PFKFB4 expression and GC in clinical samples. The current results revealed that PFKFB4 protein expression was upregulated in GC tissues compared with in adjacent normal tissues. In addition, the expression levels of PFKFB4 were upregulated in the tumor tissues from patients who were $<65$ years old compared with tissues in patients who are $\geq 65$ years old. Since PFKFB4 is a crucial enzyme involved in glucose metabolism, one of the explanations for this phenomenon may be that the metabolism in younger people is more active compared with that in older people. Moreover, patients with a tumor size $>4 \mathrm{~cm}$ or those with a high pathological stage had higher expression levels of PFKFB4, indicating PFKFB4 may be involved in the malignant progression of GC.

Next, the association between PFKFB4 expression and the prognosis of patients with GC was investigated. By comparing the differences in OS, FPS and PPS between patients with low and high PFKFB4 expression, it was discovered that high expression levels of PFKFB4 were positively associated with a decreased OS, FPS and PPS compared with low expression levels, which is consistent with the results from previous studies conducted in other types of cancer (14-16). These results suggested that PFKFB4 may be a poor prognostic indicator for patients with cancer $(26,27)$.

After determining that PFKFB4 expression was associated with a higher pathological stage in GC, the association between PFKFB4 expression and the OS of patients with GC with different $\mathrm{pT}$ or $\mathrm{pTNM}$ stages was further investigated. No significant differences were observed in the OS between patients with early stage GC and high or low PFKFB4 expression (pT2 or pTNM II); however, patients with high expression levels of PFKFB4 in the advanced stage GC group had a significantly worse OS compared with patients with low expression levels.

In conclusion, the present study revealed the expression profile of PFKFB4 in GC and analyzed its clinical significance. Since PFKFB4 expression was upregulated in GC tissues, and higher PFKFB4 expression was positively associated with a shorter survival time for patients, the current results suggested that PFKFB4 may serve as a useful poor prognostic marker for $\mathrm{GC}$, as well as an important therapeutic target for the prevention of GC progression. To the best of our knowledge, this was the first study to investigate the role of PFKFB4 in GC progression and prognosis. However, a potential limitation of the present study is the lack of follow-up of the patients in the current cohort, and the absence of survival data including these patients. Most of the patients in the cohort were from the mountain regions of western China, a vast under-populated land with relatively low levels of economic development, making it difficult to follow up these patients. In the present study, only the KM plotter database was used to collect information on the clinical significance of PFKFB4. In addition, further studies are required to elucidate the function and underlying mechanism of PFKFB4 in GC using in vitro and/or in vivo experiments.

\section{Acknowledgements}

Not applicable. 


\section{Funding}

The present study was supported by grants from the Ningxia Natural Science Foundation (grant no. NZ15150) and the National Natural Science Foundation of China (grant nos. 81660486, 81872395 and 81860442).

\section{Availability of data and materials}

The datasets used and/or analyzed during the current study are available from the corresponding author on reasonable request.

\section{Authors' contributions}

FW and YG participated in the study design. XW, YL and $\mathrm{CZ}$ assisted in collecting the specimens and IHC staining. FW, XC, YG and YL performed the data analysis. YG and $\mathrm{XW}$ drafted the manuscript. The authenticity of all the raw data was assessed by FW and YG to ensure its legitimacy. All authors provided critical review of the manuscript and read and approved the final manuscript.

\section{Ethics approval and consent to participate}

The study was approved by the Medical Research Ethics Review Committee of the General Hospital of Ningxia Medical University (Yinchuan, China). Oral informed consent from patients was obtained before collecting specimens.

\section{Patient consent for publication}

Not applicable.

\section{Competing interests}

The authors declare that they have no competing interests.

\section{References}

1. Bray F, Ferlay J, Soerjomataram I, Siegel RL, Torre LA and Jemal A: Global cancer statistics 2018: GLOBOCAN estimates of incidence and mortality worldwide for 36 cancers in 185 countries. CA Cancer J Clin 68: 394-424, 2018.

2. Cheng C, Zhang Q, Zhuang L and Sun J: Prognostic value of lymphocyte-to-C-reactive protein ratio in patients with gastric cancer after surgery: A multicentre study. Jpn J Clin Oncol 50: 1141-1149, 2020

3. Maeda K, Shibutani M, Otani H, Nagahara H, Ikeya T, Iseki Y, Tanaka H, Muguruma K and Hirakawa K: Inflammation-based factors and prognosis in patients with colorectal cancer. World J Gastrointest Oncol 7: 111-117, 2015.

4. Liu H, Zhao YR, Chen B, Ge Z and Huang JS: High expression of SMARCE1 predicts poor prognosis and promotes cell growth and metastasis in gastric cancer. Cancer Manag Res 11: 3493-3509, 2019.

5. Zhang XG, Song BT, Liu FJ, Sun D, Wang KX and Qu H: CCR6 overexpression predicted advanced biological behaviors and poor prognosis in patients with gastric cancer. Clin Transl Oncol 18: 700-707, 2016.

6. Koppenol WH, Bounds PL and Dang CV: Otto Warburg's contributions to current concepts of cancer metabolism. Nat Rev Cancer 11: 325-327, 2011.

7. Lai W, Wang Z and Liang HJ: Expression of 6-phosphofructokinase-2 in colorectal cancer and its clinical significance. J Third Military Med University (Chinese) 39: 983-989, 2017.

8. Chesney J, Clark J, Klarer AC, Imbert-Fernandez Y, Lane AN and Telang S: Fructose-2,6-bisphosphate synthesis by 6-phosphofructo-2-kinase/fructose-2,6-bisphosphatase 4 (PFKFB4) is required for the glycolytic response to hypoxia and tumor growth. Oncotarget 5: 6670-6686, 2014.
9. Li XM and Li W: Progress in the study on the role of PFKFB4 and potential mechanism in tumor glucose metabolism. Chin J N Clin Med 11: 1044-1048, 2018.

10. Li W, Qian L, Lin J, Huang G, Hao N, Wei X, Wang W and Liang J: CD44 regulates prostate cancer proliferation, invasion and migration via PDK1 and PFKFB4. Oncotarget 8: 65143-65151, 2017

11. Wang M and Sun TY: Effects of PFKFB4 on invasion and migration of gastric cancer cells and its mechanism. J Pract Oncol (Chinese) 34: 229-234, 2020.

12. Washington K: 7th Edition of the AJCC cancer staging manual: Stomach. Ann Surg Oncol 17: 3077-3079, 2010.

13. Xu L and Yang WT: The judgement standard of immunohistochemical reaction results. Chin Oncol 6: 229-231, 1996.

14. Gao R, Liu Y, Li D, Xun J, Zhou W, Wang P, Liu C, Li X, Shen W, $\mathrm{Su} \mathrm{W}$, et al: PFKFB4 promotes breast cancer metastasis via induction of hyaluronan production in a $\mathrm{p} 38$-Dependent manner. Cell Physiol Biochem 50: 2108-2123, 2018.

15. Li X, Chen Z, Li Z, Huang G, Lin J, Wei Q, Liang J and Li W: The metabolic role of PFKFB4 in androgen-independent growth in vitro and PFKFB4 expression in human prostate cancer tissue. BMC Urol 20: 61, 2020.

16. Zhang H, Lu C, Fang M, Yan W, Chen M, Ji Y, He S, Liu T, Chen T and Xiao J: HIF-1 $\alpha$ activates hypoxia-induced PFKFB4 expression in human bladder cancer cells. Biochem Biophys Res Commun 476: 146-152, 2016

17. Lu H, Chen S, You Z, Xie C, Huang S and Hu X: PFKFB4 negatively regulated the expression of histone acetyltransferase GCN5 to mediate the tumorigenesis of thyroid cancer. Dev Growth Differ 62: 129-138, 2020.

18. Chesney J, Clark J, Lanceta L, Trent JO and Telang S: Targeting the sugar metabolism of tumors with a first-in-class 6-phosphofructo-2-kinase (PFKFB4) inhibitor. Oncotarget 6: 18001-18011, 2015.

19. Minchenko OH, Tsuchihara K, Minchenko DO, Bikfalvi A and Esumi H: Mechanisms of regulation of PFKFB expression in pancreatic and gastric cancer cells. World J Gastroenterol 20: 13705-13717, 2014

20. Ros S, Floter J, Kaymak I, Da Costa C, Houddane A, Dubuis S, Griffiths B, Mitter R, Walz S, Blake S, et al: 6-Phosphofructo-2-kinase/fructose-2,6-biphosphatase 4 is essential for p53-null cancer cells. Oncogene 36: 3287-3299, 2017.

21. Shu Y, Lu Y, Pang X, Zheng W, Huang Y, Li J, Ji J, Zhang C and Shen P: Phosphorylation of PPARg at Ser84 promotes glycolysis and cell proliferation in hepatocellular carcinoma by targeting PFKFB4. Oncotarget 7: 76984-76994, 2016.

22. Gao R, Li D, Xun J, Zhou W, Li J, Wang J, Liu C, Li X, Shen W, Qiao H, et al: CD44ICD promotes breast cancer stemness via PFKFB4-mediated glucose metabolism. Theranostics 8: 6248-6262, 2018.

23. Trojan SE, Piwowar M, Ostrowska B, Laidler $P$ and Kocemba-Pilarczyk KA: Analysis of malignant melanoma cell lines exposed to hypoxia reveals the importance of PFKFB4 overexpression for disease progression. Anticancer Res 38: 6745-6752, 2018

24. Wang Q, Zeng F, Sun Y, Qiu Q, Zhang J, Huang W, Huang J, Huang $X$ and Guo L: Etk interaction with PFKFB4 modulates chemoresistance of small-cell lung cancer by regulating autophagy. Clin Cancer Res 24: 950-962, 2018.

25. Dasgupta S, Rajapakshe K, Zhu B, Nikolai BC, Yi P, Putluri N, Choi JM, Jung SY, Coarfa C, Westbrook TF, et al: Metabolic enzyme PFKFB4 activates transcriptional coactivator SRC-3 to drive breast cancer. Nature 556: 249-254, 2018.

26. Yao L, Wang L, Cao ZG, Hu X and Shao ZM: High expression of metabolic enzyme PFKFB4 is associated with poor prognosis of operable breast cancer. Cancer Cell Int 19: 165, 2019.

27. Yun SJ, Jo SW, Ha YS, Lee OJ, Kim WT, Kim YJ, Lee SC and Kim WJ: PFKFB4 as a prognostic marker in non-muscle-invasive bladder cancer. Urol Oncol 30: 893-899, 2012.

This work is licensed under a Creative Commons Attribution-NonCommercial-NoDerivatives 4.0 International (CC BY-NC-ND 4.0) License. 